\title{
Inferring Entropy Production from Short Experiments
}

\author{
Sreekanth K. Manikandan $\odot,{ }^{1}$ Deepak Gupta $\odot,{ }^{2}$ and Supriya Krishnamurthy ${ }^{1}$ \\ ${ }^{1}$ Department of Physics, Stockholm University, SE-10691 Stockholm, Sweden \\ ${ }^{2}$ Dipartimento di Fisica “G. Galilei,” INFN, Università di Padova, Via Marzolo 8, 35131 Padova, Italy
}

(Received 9 October 2019; accepted 18 February 2020; published 23 March 2020)

\begin{abstract}
We provide a strategy for the exact inference of the average as well as the fluctuations of the entropy production in nonequilibrium systems in the steady state, from the measurements of arbitrary current fluctuations. Our results are built upon the finite-time generalization of the thermodynamic uncertainty relation, and require only very short time series data from experiments. We illustrate our results with exact and numerical solutions for two colloidal heat engines.
\end{abstract}

DOI: 10.1103/PhysRevLett.124.120603

A fundamental property of nonequilibrium systems is the existence of currents which are fueled by a nonvanishing average rate of total entropy production $\sigma=\left\langle\Delta S_{\text {tot }}\right\rangle / \tau$, where $\tau$ is the time interval over which we observe the system. An estimate of $\sigma$ quantifies how much heat is dissipated to the environment on average or how much free energy is lost per unit time. More information is available from fluctuations of $\Delta S_{\text {tot }}$. These are governed by the fluctuation theorems [1-12] and can be used for the estimation of free energy differences $[13,14]$ and studying the binding energies in single-molecule $[15,16]$ or multimolecular experiments [17]. An accurate quantification of the statistics of $\Delta S_{\text {tot }}$ could also help improve our understanding of the nonequilibrium physics of active matter systems [18], biological systems [19,20], and nanoscale devices [21-24] such as colloidal heat engines [25,26].

The main challenge in the thermodynamic characterization of microscopic systems continues to be, however, the lack of a general scheme for the measurement and characterization of $\Delta S_{\text {tot }}$. For systems such as colloidal particles, for which the full dynamical equations are known, stochastic thermodynamics provides a framework to quantify $\Delta S_{\text {tot }}$ from individual trajectories $[11,27,28]$. For more complex systems where not all relevant mesostates are accessible, these direct strategies fail $[29,30]$. The only options are either to perform local calorimetric measurements to directly measure the heat emitted to the bath [31] or to come up with a new scheme for inferring $\sigma$ indirectly.

Recently, for nonequilibrium systems in a steady state, such a scheme for identifying $\sigma$ has been proposed [29,32]

Published by the American Physical Society under the terms of the Creative Commons Attribution 4.0 International license. Further distribution of this work must maintain attribution to the author(s) and the published article's title, journal citation, and DOI. Funded by Bibsam. using the thermodynamic uncertainty relation [33,34]. Using this scheme, a lower bound $\sigma_{L}$ for $\sigma$ can be obtained from the measurement of any fluctuating current $J$, in terms of its mean $\langle J\rangle$ and variance $\operatorname{Var}(J)$ as,

$$
\sigma \geq \sigma_{L} \equiv \frac{2 k_{B}\langle J\rangle^{2}}{\tau \operatorname{Var}(J)}
$$

Here, $k_{B}$ is the Boltzmann constant. Equation (1) holds for arbitrary $\tau$ for nonequilibrium systems in a steady state [35], and the proof follows from a $\sigma$-dependent parabolic bound on the large deviation function [36] of $J[37,38]$.

This inference scheme for $\sigma$ has been shown [32] to perform better than more direct methods that use spatial or temporal averages. However, since the uncertainty relation is an inequality, Eq. (1) still only gives a bound for $\sigma$ even when $J=\Delta S_{\text {tot }}$. How tight this bound is depends in general on model details and the $J$ chosen. As a result, there has been much interest recently on how to choose $J$ such that the bound value is the tightest [32,39]. For $\tau \rightarrow \infty$, it is known that the current $J$ that gives the best bound is $J=$ $\Delta S_{\text {tot }}[37]$.

Equation (1) could be used to predict $\sigma$ exactly if the equality was to hold. One case when this is known to happen is the equilibrium limit $[33,37,40]$ with $J=\Delta S_{\text {tot }}$. This means, that for systems working in the close-toequilibrium or linear response regimes, there is a possibility to estimate $\sigma$ arbitrarily close to the exact value by using Eq. (1). The equality in Eq. (1) is also met for arbitrary nonequilibrium conditions if along with $J=\Delta S_{\text {tot }}$, certain conditions are met by the steady state current and probability distributions $[41,42]$. However, there is no general scheme available so far for inferring sigma exactly under arbitrary nonequilibrium conditions. In addition no scheme exists, to our knowledge, for inferring fluctuations in $\Delta S_{\text {tot }}$.

We address precisely these issues in this Letter. Our first central contribution is to provide a new strategy which, in principle, can estimate $\sigma$ exactly at arbitrary 
nonequilibrium conditions, by using Eq. (1) in the $\tau \rightarrow 0$ limit. In this limit, for the current $J=\Delta S_{\text {tot }}$, it can be shown that the equality condition holds, just as for the equilibrium limit. Using this feature, we show that we can infer $\sigma$ arbitrarily close to the exact value, by evaluating $\sigma_{L}$ for a variety of $J$ calculated over very short time durations, and then choosing the largest value of $\sigma_{L}$ that results. A particularly appealing point from an experimental perspective is that, since we need to only compute $\sigma_{L}$ of Eq. (1) over very short trajectories, a single long time series could suffice to obtain both $\langle J\rangle$ and $\operatorname{Var}(J)$. Notice that the value of $\sigma$ so inferred is the steady-state value and hence valid at all times.

Our second contribution is to demonstrate that, by combining the value of $\sigma$ inferred from the previous step and the structure of the large deviation function of arbitrary currents $[37,38,43]$, we can also infer the distribution of $\Delta S_{\text {tot }}$, and as a result all the cumulants, arbitrarily close to their exact values. We illustrate all our findings using exact and numerical solutions for two models of colloidal engines, namely, the Brownian gyrator $[22,44]$ as well as the isothermal work-to-work converter engine [45].

We begin by considering the uncertainty relation for $J=\Delta S_{\text {tot }}$, which reads (setting $k_{B}=1$ ),

$$
\frac{\operatorname{Var}\left(\Delta S_{\text {tot }}\right)}{\left\langle\Delta S_{\text {tot }}\right\rangle} \geq 2
$$

To motivate that this inequality saturates at $\tau \rightarrow 0$, we consider the arbitrary time, scaled cumulant generating function (SCGF) $\phi_{\Delta S_{\text {tot }}}(\lambda, \tau) \equiv(1 / \tau) \log \left\langle e^{-\lambda \Delta S_{\text {tot }}}\right\rangle_{\tau}$. For short time durations, when $\left|\Delta S_{\text {tot }}\right| \ll 1$, we can express $\phi_{\Delta S_{\text {tot }}}(\lambda, \tau)$ as a series expansion in terms of the cumulants of $\Delta S_{\text {tot }}$. Then, to the leading order that respects convexity, we get (see the Supplemental Material [46] for more details and explicit expressions for the first four cumulants for an exactly solvable model)

$$
\phi_{\Delta S_{\mathrm{tot}}}(\lambda, \tau) \sim-\frac{\lambda\left\langle\Delta S_{\mathrm{tot}}\right\rangle}{\tau}+\frac{\lambda^{2} \operatorname{Var}\left(\Delta S_{\mathrm{tot}}\right)}{2 \tau} .
$$

Now applying the integral fluctuation theorem [12] $\phi_{\Delta S_{\text {tot }}}(1, \tau)=0$, we get,

$$
\frac{\operatorname{Var}\left(\Delta S_{\text {tot }}\right)}{\left\langle\Delta S_{\text {tot }}\right\rangle} \rightarrow 2 \quad \text { as } \tau \rightarrow 0 .
$$

We stress that the limit $\tau \rightarrow 0$ is crucial for Eqs. (3) and (4) to hold. For an arbitrary $\tau$ they are valid only if the distribution of $\Delta S_{\text {tot }}$ is a Gaussian. A more rigorous proof is provided in Ref. [47] to the effect that the equality in Eq. (2) is always attained when $\Delta S_{\text {tot }} \rightarrow 0$. However, note for our purposes, that this happens not only for the equilibrium limit but also when $\tau \rightarrow 0$. A model which can be solved exactly for the lhs of Eq. (2) has also been shown [40] to display this behavior as $\tau \rightarrow 0$.

We now demonstrate the usefulness of Eq. (4) for inferring $\sigma$ for two nontrivial models of colloidal engines, the Brownian gyrator model $[22,44,48]$ and the work-towork converter engine [22,45], in both of which the working substance is a single colloidal particle. In the first case, the particle is in contact with external reservoirs at hot $\left(T_{1}\right)$ and cold $\left(T_{2}\right)$ temperatures and in the second case, the particle is subjected to two white-noise forces, interpreted as a load and drive force. In these models, there is a clear division of $\Delta S_{\text {tot }}$ in terms of time-extensive quantities such as heat $(Q)$ and work $(W)$ and time-intensive contributions such as the entropy change of the system $\Delta S_{\text {sys }}$. Hence $\Delta S_{\text {tot }}$ can be written as

$$
\Delta S_{\mathrm{tot}}=\frac{\eta_{C}}{T_{2}} Q_{1}+\frac{1}{T_{2}} W+\Delta S_{\mathrm{int}}
$$

Here $\eta_{C}=1-\left(T_{2} / T_{1}\right)$ is the Carnot efficiency.

The term $\Delta S_{\text {int }}=-\left(1 / T_{2}\right) \Delta E+\Delta S_{\text {sys }}$ collects the timeintensive contributions to the total entropy production that depend only on the initial and final states of the system. $\Delta E$ denotes the change in internal energy, which is, according to the First Law of thermodynamics, $\Delta E=W+Q_{1}+Q_{2}$. We define an arbitrary current $J$ in the system as the linear combination $J=c_{1}\left(\eta_{C} / T_{2}\right) Q_{1}+c_{2}\left(1 / T_{2}\right) W+c_{3} \Delta S_{\text {int }}$, where $c_{1}, c_{2}$, and $c_{3}$ are random real numbers, taken uniformly from the interval $[-1,1]$. In particular, when $c_{1}=c_{2}=c_{3}=1$, we get $J=\Delta S_{\text {tot }}$ [49].

In Fig. 1 we illustrate this inference scheme for the Brownian gyrator (Fig. 1, left panel) and the work-to-work converter engine (Fig. 1, right panel). Since both of these models have been extensively studied in the literature, we relegate their detailed descriptions to the Supplemental Material [46]. The Brownian gyrator can be solved exactly [22] for the full SCGF $\Phi\left(\lambda_{Q}, \lambda_{w}, \lambda_{S}, \tau\right) \equiv$ $(1 / \tau) \log \left\langle e^{-\lambda_{Q} Q_{1}-\lambda_{W} W+\lambda_{S} \Delta S_{\text {int }}}\right\rangle_{\tau}$ at arbitrary times and hence provides us with the means to check the inference procedure analytically (see the Supplemental Material [46], which also contains the Refs. [50-54]). The second model of the work-to-work converter can only be solved for large times [45]. We hence use it to test our inference scheme in a situation where we can only rely on numerics.

In the left panel of Fig. 1, we compute $\sigma_{L}$ for the Brownian gyrator, using our analytical solutions (see Ref. [46] for more details) for arbitrary currents $J$ at any time $\tau$. The exact value of $\sigma$ is marked by the black horizontal line. In the $\tau \rightarrow 0$ limit, $\Delta S_{\text {tot }}$ infers $\sigma$ exactly. Also, notice that there are other currents which perform almost as good as $\Delta S_{\text {tot }}$, and infer $\sigma$ arbitrarily close to the actual value, in the $\tau \rightarrow 0$ limit. The red solid lines correspond to a value of $\sigma_{L}$ computed from currents for which $c_{3} \neq 0$. The blue dotted lines correspond to $\sigma_{L}$ calculated from currents for which $c_{3}=0$ and hence which 

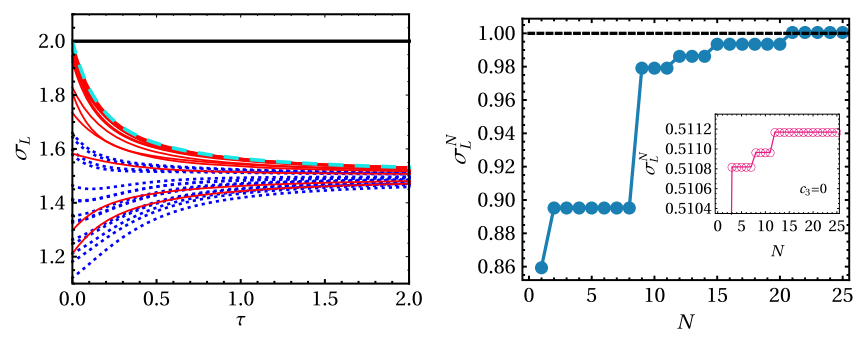

FIG. 1. An illustration of the exact estimation of the entropy production rate $\sigma$, using the $\tau \rightarrow 0$ limit of Eq. (1) for two colloidal engine models in nonequilibrium steady states. Left: $\sigma$ inferred as a function of time, for the Brownian gyrator model, with analytic solutions for $\sigma_{L}$ in Eq. (1). The black horizontal line corresponds to the actual entropy production rate. The red solid and blue dotted lines correspond to arbitrary currents with and without boundary contributions (see the main text). The results show that the best inference of $\sigma$ is given by $\Delta S_{\text {tot }}$ (green dashed line) itself, and that several of the currents having boundary contributions (red solid lines) infer $\sigma$ arbitrarily close to the actual value, in the $\tau \rightarrow 0$ limit. Right: Inferring $\sigma$, as the maximum of the measured $\sigma_{L}$ 's of $N$ arbitrary currents [Eq. (6)], for the isothermal work-to-work converter engine, from numerical simulations. Here the black dashed line corresponds to the actual $\sigma$, obtainable from a large-time computation [45]. $\sigma_{L}^{N}$ corresponds to the maximum $\sigma_{L}$ inferred by the $N$ currents, at $\tau \rightarrow 0$. We see that as $N$ increases, $\sigma_{L}^{N}$ saturates to the known value of $\sigma$. The inset shows that the inference procedure makes a large error if the boundary terms are not included.

are only linear combinations of $Q_{1}$ and $W$, the timeextensive contributions to $\Delta S_{\text {tot }}$. The best inference strategy is therefore to measure the mean and variance of an ensemble of randomly generated currents, at short times. Since, the bound in Eq. (1) saturates for $\tau \rightarrow 0$, we are guaranteed to obtain a value for $\sigma_{L}$ arbitrarily close to the actual $\sigma$ as

$$
\sigma=\max _{J}\left\{\lim _{\tau \rightarrow 0} \sigma_{L}\right\}
$$

Note that for large $\tau$ all currents, including $J=\Delta S_{\text {tot }}$, give a similar estimate, which is considerably less than the actual value (left panel, Fig. 1). Hence, the small-time saturation of Eq. (1) as well as it's sensitivity to the chosen $J$, both work in favor of getting a better estimate for $\sigma$ than at large $\tau$. In practice, the $\tau \rightarrow 0$ limit may be achieved in experiments by choosing trajectory lengths corresponding to the minimal temporal resolution accessible to the experiment $[55,56]$ such that $\Delta S_{\text {tot }}$ also becomes arbitrarily small in this limit. In the right panel of Fig. 1, we numerically compute $\sigma_{L}$ for the second model of the work-to-work converter, by computing the mean and variance of different randomly chosen $J$ for very short trajectories and using Eq. (6). Since this model can be solved in the steady state [45], the exact value of $\sigma$ is known. As can be seen, the inferred value is in very good agreement with the exact value after the inference procedure has been applied to the order of about 20 currents.

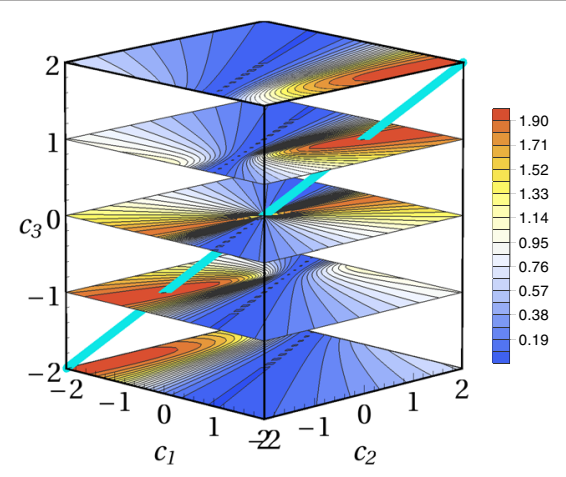

FIG. 2. Entropy production rate inferred in the $\tau \rightarrow 0$ limit, for the Brownian gyrator model. Arbitrary currents are constructed as linear combinations of the basis currents $Q_{1}, W$, and $\Delta S_{\text {int }}$, with coefficients $c_{1}, c_{2}$, and $c_{3}$. The green line corresponds to the $\Delta S_{\text {tot }}$ current direction. In the scale provided, red corresponds to a more accurate inference and blue to an inaccurate inference. It is found that, for nonzero values of $c_{3}$, the best inference is given by the $\Delta S_{\text {tot }}$ current itself. However, the optimal current differs from the corresponding $\Delta S_{\text {tot }}$, when we set $c_{3}=0$ (see Supplemental Material [46]).

To further analyze the inference of $\sigma$ by the short-time inference scheme, Eq. (6), we have identified the optimal currents that infer $\sigma$ the best. In Fig. 2, we illustrate this in the $\tau \rightarrow 0$ limit for the Brownian gyrator. When $c_{3} \neq 0$ the current that infers best is $\Delta S_{\text {tot }}$ itself, as expected. However, for $c_{3}=0$, some other direction in the $\left(c_{1}, c_{2}\right)$ plane gives the current that infers best (Fig. 2). Even when $\tau>0$, we find (see Supplemental Material [46]) that the optimal current is in general different from $\Delta S_{\text {tot }}$ as recently suggested in Ref. [39], and becomes equal to $\Delta S_{\text {tot }}$ only for large $\tau$ [37].

It is important to note that, our usage of the $c_{1}, c_{2}, c_{3}$ space is purely a proof of concept, to demonstrate the viability and applicability of our short-time inference scheme, as well as to disentangle the separate roles played by the time-intensive and time-extensive terms which would not have been obvious otherwise. In addition, in this space, currents have natural physical interpretations in terms of work, heat, and system entropy production. For a generic nonequilibrium system, however, for which neither a Langevin nor a master equation description is known, such a decomposition of $\Delta S_{\text {tot }}$ [Eq. (5)] is usually not possible. In such cases, one can generate random currents from the phase space trajectories $\mathbf{x}(t)$ of the system [32,57] using random vectors $\mathbf{d}(\mathbf{x})$ as $J \equiv J_{d}=\int_{\mathbf{x}(0)}^{\mathbf{x}(\tau)} d \mathbf{x} \mathbf{d}(\mathbf{x}) \mathbf{j}(\mathbf{x})$, where $\mathbf{j}(\mathbf{x})=(1 / \tau) \int_{0}^{\tau} \delta[\mathbf{x}-\mathbf{x}(t)] d \mathbf{x}(t)$ is an estimate for a steady state current. Such a current can then be used to compute $\sigma_{L}$ in Eq. (1) and many such currents can be generated by varying $\mathbf{d}$. Our scheme will then imply that as $\tau \rightarrow 0$, the current that will satisfy the equality in Eq. (1) will correspond to $\Delta S_{\text {tot }}$. Note that $J_{d}$ 's defined this way contain information of both the extensive and intensive contributions and there is no need to calculate the steady 
state distribution separately. In the Supplemental Material, we show the $\mathbf{d}$ field which corresponds to $J=\Delta S_{\text {tot }}$ for the Brownian gyrator. For problems where an analytical treatment is ruled out, such a d field would have to be obtained by some optimization procedure [32].

So far, we have shown that the finite time thermodynamic uncertainty relation can be used at very short observational times to infer $\sigma$ arbitrarily close to the actual value, arbitrarily far from equilibrium. It is then natural to ask, if there exist similar inference strategies for the fluctuations of $\Delta S_{\text {tot }}$ as well. Since the current that infers $\sigma$ the best at $\tau=0$ is $J=\Delta S_{\text {tot }}$, a naive solution would have been to approximate the optimal current in the $\tau \rightarrow 0$ limit by $\Delta S_{\text {tot }}$, and compute a distribution directly from that. Although this can work in principle, there are a couple of practical disadvantages. First, with the real data, one is always limited by the minimal experimental resolution $\Delta t>0$ and the $\tau \rightarrow 0$ limit can be accessed only within this error. Second, there can be several near optimal currents (as shown in Fig. 1, left) that infer $\sigma$ arbitrarily close to the exact value, which a computational scheme may not be able to distinguish. For example, it has been noticed in Ref. [32], for finite $\tau$, that these near optimal currents can look very different from each other, and still predict a similar value for $\sigma$. As a result of these limitations, because of which we may have access to a value $\sigma$, but not the current $J=\Delta S_{\text {tot }}$, we present an alternate strategy for computing the steady state distribution of $\Delta S_{\text {tot }}$ using an exact estimate of $\sigma$.

We begin by considering the structure of the scaled cumulant generating function of an arbitrary current in the steady state, $\phi_{J}^{\sigma}(\lambda, \tau) \equiv(1 / \tau) \log \left\langle e^{-\lambda(\sigma \tau J /\langle J\rangle)}\right\rangle_{\tau}$ at large $\tau$. Using large deviation techniques, it has been shown recently that $\phi_{J}^{\sigma}$ obeys the bound $[37,43]$

$$
-\sigma \lambda(1-\lambda) \leq \phi_{\Delta S_{\mathrm{tot}}}(\lambda, \tau) \leq \phi_{J}^{\sigma}(\lambda, \tau) .
$$

The uncertainty relation in Eq. (1) can be directly proved from this result $[37,38]$. Interestingly, Eq. (7) constrains the fluctuations of $\Delta S_{\text {tot }}$ strongly, by providing both a lower bound and an upper bound for $\phi_{\Delta S_{\text {tot }}}$. In particular, one can saturate the bound $\phi_{\Delta S_{\text {tot }}}(\lambda, \tau) \leq \phi_{J}^{\sigma}(\lambda, \tau)$ if $J=\Delta S_{\text {tot }}$. We therefore get a natural scheme for inferring $\phi_{\Delta S_{\text {tot }}}(\lambda, \tau)$ as

$$
\phi_{\Delta S_{\mathrm{tot}}}(\lambda, \tau)=\min _{J}\left\{\phi_{J}^{\sigma}(\lambda, \tau)\right\}
$$

Consequently, if $M_{J}^{(n)}$ is the $n$th cumulant of the normalized current $\sigma \tau J /\langle J\rangle$, we also get (see Ref. [46])

$$
M_{\Delta S_{\mathrm{tot}}}^{(n)}=\min _{J}\left\{M_{J}^{(n)}\right\} .
$$

The cumulants thus inferred can then be used to construct the histogram of $\Delta S_{\text {tot }}$ straightforwardly.

We illustrate Eqs. (8) and (9) in Fig. 3 for the isothermal work-to-work converter engine. We have first obtained an
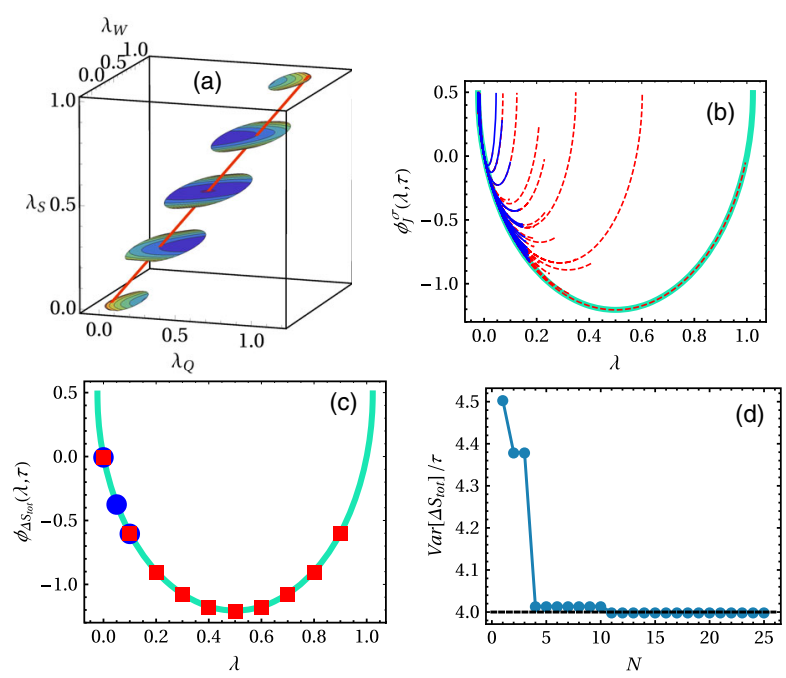

FIG. 3. Results for the isothermal work-to-work converter engine. (a) $\Phi\left(\lambda_{Q}, \lambda_{W}, \lambda_{S}, \tau\right)$ obtained analytically. $\Phi$ has a limited domain of convergence (only a few $\lambda_{S}$ planes are shown for clarity), and also displays the fluctuation theorem symmetry around the point $(0.5,0.5,0.5)$. In (b) and (c), the thick green curve is the actual $\phi_{\Delta S_{\text {tot }}}$. In (b), the blue solid and red dashed curves, respectively, correspond to $\phi_{J}^{\sigma}$ with $c_{3}=0$ and $c_{3} \neq 0$ case. In (c), $\phi_{\Delta S_{\text {tot }}}$ is obtained using Eq. (8), where red squares and blue circles, respectively, correspond to $c_{3} \neq 0$ and $c_{3}=0$ at large $\tau$ [46]. (d) Inferring $\operatorname{Var}\left(\Delta S_{\text {tot }}\right)$, as the minimum of the measured variances of $N$ scaled, arbitrary currents, $\sigma \tau J /\langle J\rangle$ according to Eq. (9). Here the black line corresponds to the variance of $\Delta S_{\text {tot }}$, in the long time limit [45].

analytic expression for the joint SCGF $\Phi\left(\lambda_{Q}, \lambda_{W}, \lambda_{S}, \tau\right)=$ $(1 / \tau) \log \left\langle e^{-\left(\lambda_{Q} \eta_{C} Q_{1} / T_{2}\right)-\left(\lambda_{W} W / T_{2}\right)-\left(\lambda_{S} \Delta S_{\text {int }}\right)}\right\rangle_{\tau}$, which is exact at large but finite times (see the Supplemental Material [46]). The geometry of $\Phi$ was recently conjectured and discussed in some detail in Ref. [22]. Because of the fluctuation theorem, $\Phi$ is a reflection symmetric object around the point $(0.5,0.5,0.5)$, and typically has a limited domain of convergence (cutoffs) that depend on $\lambda_{S}$. We illustrate this for a fixed, large value of $\tau$ in Fig. 3(a). The SCGF of an arbitrary current $J$ can be obtained from $\Phi$ by evaluating it along a straight line passing through the origin and the point $\left(c_{1}, c_{2}, c_{3}\right)$, where $c_{1}, c_{2}, c_{3}$ are random numbers. In particular, $\phi_{\Delta S_{\text {tot }}}$ is $\Phi$ evaluated along the $(1,1,1)$ direction marked by the red solid line in Fig. 3(a).

In Figs. 3(b) and 3(c), we illustrate the inference of $\phi_{\Delta S_{\text {tot }}}$ using Eq. (8). Since $\phi_{J}^{\sigma}$ of currents with $c_{3}=0$, can have restricted domains of convergences [see $\lambda_{S}=0$ plane of Fig. 3(a)], they will end up inferring a limited domain of $\phi_{\Delta S_{\text {tot }}}$, as shown in Fig. 3(c) with the blue circles. The red squares show the improvement in the estimate of $\phi_{\Delta S_{\text {tot }}}$ when $c_{3} \neq 0$. In Fig. 3(d), we illustrate the inference of $\operatorname{Var}\left(\Delta S_{\text {tot }}\right)$ using Eq. (9), numerically.

In summary, we have presented a scheme to exactly infer the average entropy production rate $\sigma$ as well as the distribution of entropy production $P\left(\Delta S_{\text {tot }}\right)$ in nonequilibrium 
steady state systems. The scheme for identifying $\sigma$ is built upon the finite time thermodynamic uncertainty relation $[35,38]$ and its saturation in the very short time limit. The inference of $P\left(\Delta S_{\text {tot }}\right)$ is then built upon an exactly estimated value of $\sigma$ and the dissipation bounded structure of steady state current fluctuations $[37,43]$.

We have chosen to demonstrate our scheme using a decomposition of $\Delta S_{\text {tot }}$ into time-extensive and intensive contributions, but we expect that our scheme is equally applicable for currents generated any other way. Such considerations will be very relevant when testing this scheme in biological systems or active matter systems in the steady state. Generalizations to time symmetrically driven systems [47] could also be interesting.

D. G. acknowledges the support from University of Padova through project "Excellence Project 2018" of the Cariparo Foundation. S. K. and S. K. M. thank Lennart Dabelow, Ralf Eichhorn, Shun Otsubo, Stefano Bo, and Takahiro Sagawa for helpful discussions. We also thank Shun Otsubo for important comments on an earlier version of the manuscript.

Note added.-Recently, Refs. [58,59] have independently found further proofs and refinements of the short-time inference scheme, and have proposed efficient computational algorithms to implement it.

[1] D. J. Evans, E. G. D. Cohen, and G. P. Morriss, Probability of Second Law Violations in Shearing Steady States, Phys. Rev. Lett. 71, 2401 (1993).

[2] D. J. Evans and D. J. Searles, Equilibrium microstates which generate second law violating steady states, Phys. Rev. E 50, 1645 (1994).

[3] D. J. Searles and D. J. Evans, Ensemble dependence of the transient fluctuation theorem, J. Chem. Phys. 113, 3503 (2000).

[4] D. J. Searles and D. J. Evans, Fluctuation theorem for heat flow, Int. J. Thermophys. 22, 123 (2001).

[5] G. Gallavotti and E. G. D. Cohen, Dynamical Ensembles in Nonequilibrium Statistical Mechanics, Phys. Rev. Lett. 74, 2694 (1995).

[6] G. Gallavotti and E. G. D. Cohen, Dynamical ensembles in stationary states, J. Stat. Phys. 80, 931 (1995).

[7] J. Kurchan, Fluctuation theorem for stochastic dynamics, J. Phys. A 31, 3719 (1998).

[8] J. L. Lebowitz and H. Spohn, A Gallavotti-Cohen-type symmetry in the large deviation functional for stochastic dynamics, J. Stat. Phys. 95, 333 (1999).

[9] C. Jarzynski, Nonequilibrium Equality for Free Energy Differences, Phys. Rev. Lett. 78, 2690 (1997).

[10] G. E. Crooks, Entropy production fluctuation theorem and the nonequilibrium work relation for free energy differences, Phys. Rev. E 60, 2721 (1999).

[11] U. Seifert, Stochastic thermodynamics, fluctuation theorems and molecular machines, Rep. Prog. Phys. 75, 126001 (2012).
[12] U. Seifert, Entropy Production Along a Stochastic Trajectory and an Integral Fluctuation Theorem, Phys. Rev. Lett. 95, 040602 (2005).

[13] G. Hummer and A. Szabo, Free energy reconstruction from nonequilibrium single-molecule pulling experiments, Proc. Natl. Acad. Sci. U.S.A. 98, 3658 (2001).

[14] J. Liphardt, S. Dumont, S. B. Smith, I. Tinoco, and C. Bustamante, Equilibrium information from nonequilibrium measurements in an experimental test of Jarzynski's equality, Science 296, 1832 (2002).

[15] A. Mossa, M. Manosas, N. Forns, J. M. Huguet, and F. Ritort, Dynamic force spectroscopy of DNA hairpins: I. Force kinetics and free energy landscapes, J. Stat. Mech. (2009) P02060.

[16] F. Ritort, Single-molecule experiments in biological physics: Methods and applications, J. Phys. Condens. Matter 18, R531 (2006).

[17] J. Camunas-Soler, A. Alemany, and F. Ritort, Experimental measurement of binding energy, selectivity, and allostery using fluctuation theorems, Science 355, 412 (2017).

[18] S. Ramaswamy, The mechanics and statistics of active matter, Annu. Rev. Condens. Matter Phys. 1, 323 (2010).

[19] M. E. Cates, Diffusive transport without detailed balance in motile bacteria: Does microbiology need statistical physics?, Rep. Prog. Phys. 75, 042601 (2012).

[20] D. S. Seara, V. Yadav, I. Linsmeier, A. P. Tabatabai, P. W. Oakes, S. M. Ali Tabei, S. Banerjee, and M. P. Murrell, Entropy production rate is maximized in non-contractile actomyosin, Nat. Commun. 9, 4948 (2018).

[21] P. Pietzonka and U. Seifert, Universal Trade-Off Between Power, Efficiency, and Constancy in Steady-State Heat Engines, Phys. Rev. Lett. 120, 190602 (2018).

[22] S. K. Manikandan, L. Dabelow, R. Eichhorn, and S. Krishnamurthy, Efficiency Fluctuations in Microscopic Machines, Phys. Rev. Lett. 122, 140601 (2019).

[23] G. Verley, M. Esposito, T. Willaert, and C. Van den Broeck, The unlikely Carnot efficiency, Nat. Commun. 5, 4721 (2014).

[24] G. Verley, T. Willaert, C. Van den Broeck, and M. Esposito, Universal theory of efficiency fluctuations, Phys. Rev. E 90, 052145 (2014).

[25] I. A. Martínez, É. Roldán, L. Dinis, and R. A. Rica, Colloidal heat engines: A review, Soft Matter 13, 22 (2017).

[26] I. A. Martínez, É. Roldán, L. Dinis, D. Petrov, J. M. R. Parrondo, and R. A. Rica, Brownian Carnot engine, Nat. Phys. 12, 67 (2016).

[27] K. Sekimoto, Kinetic characterization of heat bath and the energetics of thermal ratchet models, J. Phys. Soc. Jpn. 66, 1234 (1997).

[28] K. Sekimoto, Langevin equation and thermodynamics, Prog. Theor. Phys. Suppl. 130, 17 (1998).

[29] U. Seifert, From stochastic thermodynamics to thermodynamic inference, Annu. Rev. Condens. Matter Phys. 10, 171 (2019).

[30] I. A. Martínez, G. Bisker, J. M. Horowitz, and J. M. R. Parrondo, Inferring broken detailed balance in the absence of observable currents, Nat. Commun. 10, 3542 (2019).

[31] L. Basta, S. Veronesi, Y. Murata, Z. Dubois, N. Mishra, F. Fabbri, C. Coletti, and S. Heun, A sensitive calorimetric technique to study energy (heat) exchange at the nano-scale, Nanoscale 10, 10079 (2018). 
[32] J. Li, J. M. Horowitz, T. R. Gingrich, and N. Fakhri, Quantifying dissipation using fluctuating currents, Nat. Commun. 10, 1666 (2019).

[33] A. C. Barato and U. Seifert, Thermodynamic Uncertainty Relation for Biomolecular Processes, Phys. Rev. Lett. 114, 158101 (2015).

[34] A. C. Barato and U. Seifert, Universal bound on the fano factor in enzyme kinetics, J. Phys. Chem. B 119, 6555 (2015).

[35] P. Pietzonka, F. Ritort, and U. Seifert, Finite-time generalization of the thermodynamic uncertainty relation, Phys. Rev. E 96, 012101 (2017).

[36] H. Touchette, The large deviation approach to statistical mechanics, Phys. Rep. 478, 1 (2009).

[37] T. R. Gingrich, J. M. Horowitz, N. Perunov, and J. L. England, Dissipation Bounds All Steady-State Current Fluctuations, Phys. Rev. Lett. 116, 120601 (2016).

[38] J. M. Horowitz and T. R. Gingrich, Proof of the finite-time thermodynamic ivcertainty relation for steady-state currents, Phys. Rev. E 96, 020103 (2017).

[39] D. M. Busiello and S. Pigolotti, Hyperaccurate currents in stochastic thermodynamics, Phys. Rev. E 100, 060102 (2019).

[40] S. K. Manikandan and S. Krishnamurthy, Exact results for the finite time thermodynamic uncertainty relation, J. Phys. A 51, 11LT01 (2018).

[41] Y. Hasegawa and T. Van Vu, Uncertainty relations in stochastic processes: An information inequality approach, Phys. Rev. E 99, 062126 (2019).

[42] C. Hyeon and W. Hwang, Physical insight into the thermodynamic uncertainty relation using brownian motion in tilted periodic potentials, Phys. Rev. E 96, 012156 (2017).

[43] P. Pietzonka, A. C. Barato, and U. Seifert, Universal bounds on current fluctuations, Phys. Rev. E 93, 052145 (2016).

[44] R. Filliger and P. Reimann, Brownian Gyrator: A Minimal Heat Engine on the Nanoscale, Phys. Rev. Lett. 99, 230602 (2007).

[45] D. Gupta and S. Sabhapandit, Stochastic efficiency of an isothermal work-to-work converter engine, Phys. Rev. E 96, 042130 (2017).
[46] See Supplemental Material at http://link.aps.org/ supplemental/10.1103/PhysRevLett.124.120603 for details of the results and explicit calculations.

[47] Y. Hasegawa and T. Van Vu, Fluctuation Theorem Uncertainty Relation, Phys. Rev. Lett. 123, 110602 (2019).

[48] A. Argun, J. Soni, L. Dabelow, S. Bo, G. Pesce, R. Eichhorn, and G. Volpe, Experimental realization of a minimal microscopic heat engine, Phys. Rev. E 96, 052106 (2017).

[49] In principle, one can also consider any arbitrary decomposition of $\Delta S_{\text {tot }}$ with more components. We will stick to a three dimensional basis for simplicity.

[50] S. K. Manikandan and S. Krishnamurthy, Asymptotics of work distributions in a stochastically driven system, Eur. Phys. J. B 90, 258 (2017).

[51] V. Y. Chernyak, M. Chertkov, and C. Jarzynski, Pathintegral analysis of fluctuation theorems for general Langevin processes, J. Stat. Mech. (2006) P08001.

[52] L. Onsager and S. Machlup, Fluctuations and irreversible processes, Phys. Rev. 91, 1505 (1953).

[53] S. Machlup and L. Onsager, Fluctuations and irreversible process. II. Systems with kinetic energy, Phys. Rev. 91, 1512 (1953).

[54] K. Kirsten and A. J. McKane, Functional determinants by contour integration methods, Ann. Phys. (Amsterdam) 308, 502 (2003).

[55] S. Kheifets, A. Simha, K. Melin, T. Li, and M. G. Raizen, Observation of Brownian motion in liquids at short times: Instantaneous velocity and memory loss, Science 343, 1493 (2014).

[56] C. Di Rienzo, V. Piazza, E. Gratton, F. Beltram, and F. Cardarelli, Probing short-range protein brownian motion in the cytoplasm of living cells, Nat. Commun. 5, 5891 (2014).

[57] T. R. Gingrich, G. M. Rotskoff, and J. M. Horowitz, Inferring dissipation from current fluctuations, J. Phys. A 50, 184004 (2017).

[58] S. Otsubo, S. Ito, A. Dechant, and T. Sagawa, Estimating entropy production by machine learning of short-time fluctuating currents, arXiv:2001.07460.

[59] T. Van Vu, V. Tuan Vo, and Y. Hasegawa, Entropy production estimation with optimal current, arXiv:2001.07131. 